\title{
Acceleration and Storage of Polarized Proton Beam at RHIC *
}

\author{
Thomas Roser \\ AGS Department, Brookhaven National Laboratory \\ Upton, NY 11973-5000
}

\begin{abstract}
High energy polarized beam collisions will open up the unique physics opportunities of studying spin effects in hard processes. However, the acceleration of polarized beams in circular accelerators is complicated by the numerous depolarizing spin resonances. Using a partial Siberian Snake and a rf dipole that ensure stable adiabatic spin motion during acceleration has made it possible to accelerate polarized protons to $25 \mathrm{GeV}$ at the Brookhaven AGS. Full Siberian Snakes and polarimeters are being developed for RHIC to make the acceleration of polarized protons to $250 \mathrm{GeV}$ possible. Spin rotators around two RHIC interaction regions will allow for the collision of longitudinally polarized protons at the STAR and PHENIX detectors.
\end{abstract}

\section{INTRODUCTION}

Polarized proton colliders will open up the completely unique physics opportunities of studying spin effects in hard processes at high luminosity, high energy proton-proton collisions. It will allow to study the spin structure of the proton, in particular the degree of polarization of the gluons and antiquarks, and also to verify the many well documented expectations of spin effects in perturbative QCD and parity violation in $\mathrm{W}$ and $\mathrm{Z}$ production.

Proton-proton collisions at high energies involve hard scattering of gluons and quarks as is shown schematically in Fig. 1. In this kinematic region factorization should hold and any asymmetry $A$ measured for a high $p_{T}$ reaction is a sum of corresponding asymmetries $\hat{a}$ at the parton level weighted by the actual degree of polarization of the initial partons given by the spin structure functions:

$$
A=\sum_{\text {subprocesses }} \frac{\Delta a}{a} \times \frac{\Delta b}{b} \times \widehat{a}(a+b \rightarrow c+d)
$$

The subprocess asymmetries are predicted by the standard model and are often large. For example, $\widehat{a}_{L L}$ in QCD is $50 \%$ or larger for most subprocesses and the

*) Work performed under the auspices of the U.S. Department of Energy 


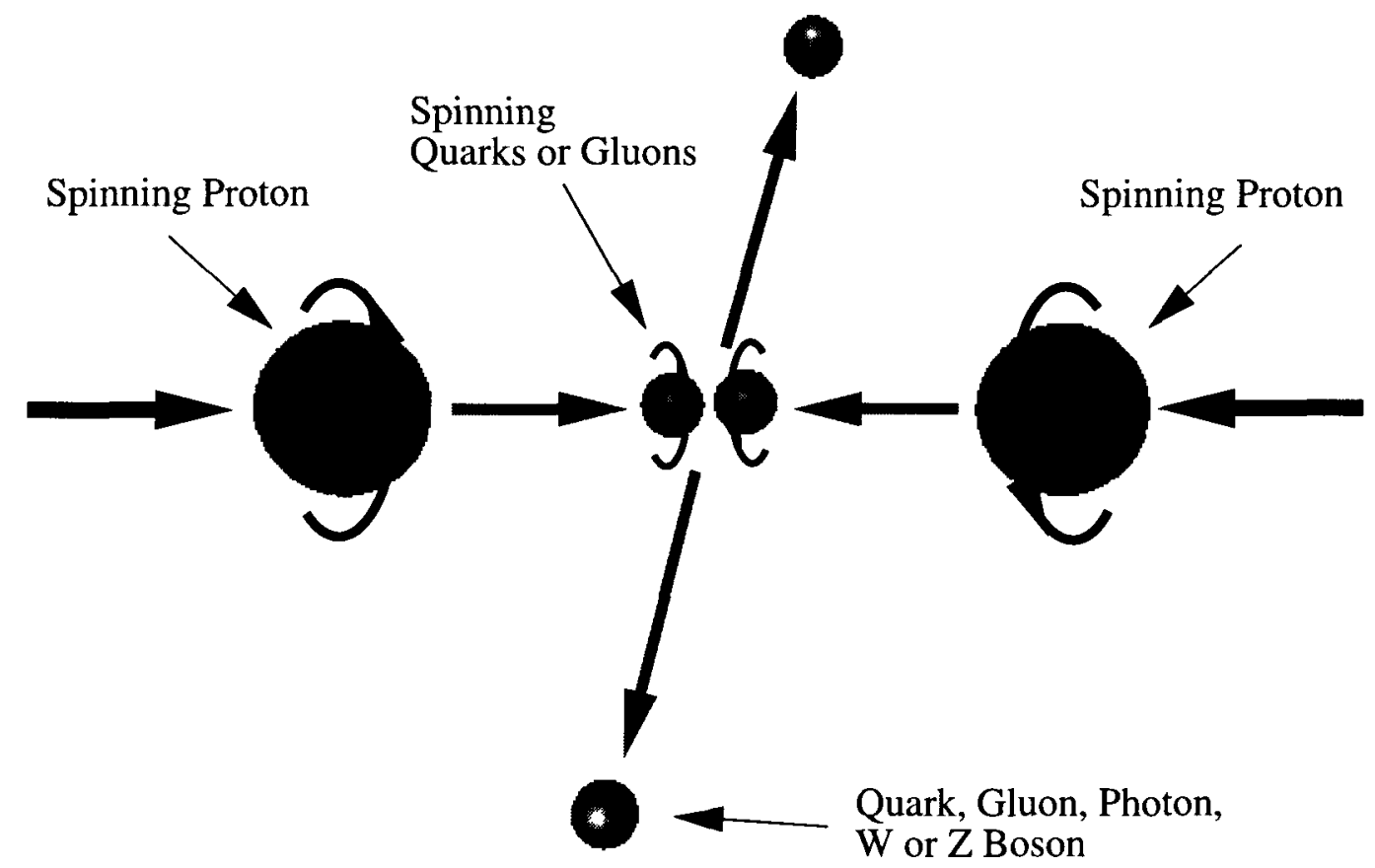

FIGURE 1. Schematic of parton collisions in high energy polarized proton collisions

parity violating $\hat{a}_{L}$ is unity in weak processes. By measuring different reactions and different types of asymmetries we can determine subprocess asymmetries, the spin structure functions of all partons, and also perform self consistency checks. This very ambitious program is greatly simplified by the fact that the spin structure functions of valence quarks are known from deep inelastic scattering measurements and that we can select reactions that are dominated by just one subprocess. A center-of-mass energy range of 200 to $500 \mathrm{GeV}$, as achievable in the Brookhaven Relativistic Heavy Ion Collider (RHIC) [1], is ideal in the sense that it is high enough for perturbative QCD to be applicable and low enough so that the average $x$ value is about 0.1 or larger which guarantees significant levels of polarization for the valence quarks.

\section{SPIN DYNAMICS, RESONANCES AND SIBERIAN SNAKES}

Accelerating polarized beams requires an understanding of both the orbital motion and spin motion. Whereas the effect of the spin on the orbit is negligible the effect of the orbit on the spin is usually very strong. The evolution of the spin direction of a beam of polarized protons in external magnetic fields such as exist in a circular accelerator is governed by the Thomas-BMT equation [2], 


$$
\frac{d \vec{P}}{d t}=-\left(\frac{e}{\gamma m}\right)\left[G \gamma \overrightarrow{B_{\perp}}+(1+G) \overrightarrow{B_{\|}}\right] \times \vec{P}
$$

where the polarization vector $P$ is expressed in the frame that moves with the particle. This simple precession equation is very similar to the Lorentz force equation which governs the evolution of the orbital motion in an external magnetic field:

$$
\frac{d \vec{v}}{d t}=-\left(\frac{e}{\gamma m}\right)\left[\overrightarrow{B_{\perp}}\right] \times \vec{v} .
$$

From comparing these two equations it can readily be seen that, in a pure vertical field, the spin rotates $G \gamma$ times faster than the orbital motion. Here $G=1.7928$ is the anomalous magnetic moment of the proton and $\gamma=E / m$. In this case the factor $G \gamma$ then gives the number of full spin precessions for every full revolution, a number which also called the spin tune $\nu_{s p}$. At top RHIC energies this number reaches about 400 . The Thomas-BMT equation also shows that at low energies $(\gamma \approx 1)$ longitudinal fields $\overrightarrow{B_{\|}}$can be quite effective in manipulating the spin motion, but at high energies transverse fields $\overrightarrow{B_{\perp}}$ need to be used to have any effect beyond the always present vertical holding field.

The acceleration of polarized beams in circular accelerators is complicated by the presence of numerous depolarizing spin resonances. During acceleration, a spin resonance is crossed whenever the spin precession frequency equals the frequency with which spin-perturbing magnetic fields are encountered. There are two main types of spin resonances corresponding to the possible sources of such fields: imperfection resonances, which are driven by magnet errors and misalignments, and intrinsic resonances, driven by the focusing fields.

The resonance conditions are usually expressed in terms of the spin tune $\nu_{s p}$. For an ideal planar accelerator, where orbiting particles experience only the vertical guide field, the spin tune is equal to $G \gamma$, as stated earlier. The resonance condition for imperfection depolarizing resonances arise when $\nu_{s p}=G \gamma=n$, where $n$ is an integer. Imperfection resonances are therefore separated by only $523 \mathrm{MeV}$ energy steps. The condition for intrinsic resonances is $\nu_{s p}=G \gamma=k P \pm \nu_{y}$, where $k$ is an integer, $\nu_{y}$ is the vertical betatron tune and $P$ is the superperiodicity. For example at the AGS, $P=12$ and $\nu_{y} \approx 8.8$.

Close to a spin resonance the spin tune deviates away from its value of $G \gamma$ of the ideal flat machine. For a resonance with strength $\epsilon$, which is the total spin rotation due to the resonance driving fields, the new spin tune is given by the equation

$$
\cos \left(\pi \nu_{s p}\right)=\cos (\pi G \gamma) \cos (\pi \epsilon)
$$

Fig. 2 shows the solutions of this equation with and without a resonance. A similar calculation can be done for the effective precession direction or, as it is now often called, the stable spin direction. The stable spin direction describes 


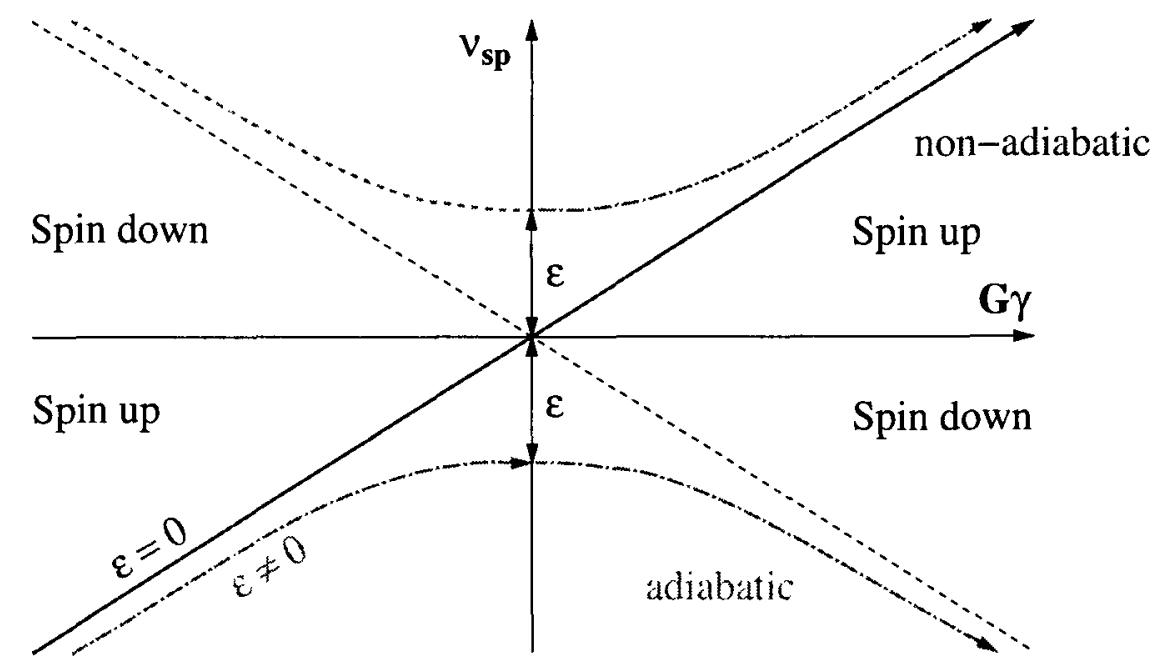

FIGURE 2. The evolution of the spin tune during the crossing of a resonance with strength $\epsilon$

those polarization components that are repeated every turn. Note that both the stable spin direction and the spin tune are completely determined by the magnetic structure of the accelerator and the beam energy. The magnitude and sign of the beam polarization, however, depends on the beam polarization at injection and the history of the acceleration process.

The spin tune and stable spin direction calculations apply only to a timeindependent static situation or if parameters are changed adiabatically. Far from the resonance the stable spin direction coincides with the main vertical magnetic field. Close to the resonance, the stable spin direction is perturbed away from the vertical direction by the resonance driving fields. When a polarized beam is accelerated through an isolated resonance at arbitrary speed, the final polarization can be calculated analytically [3] and is given by

$$
P_{f} / P_{i}=2 e^{-\frac{\pi|\epsilon|^{2}}{2 \alpha}}-1
$$

where $P_{i}$ and $P_{f}$ are the polarizations before and after the resonance crossing, respectively, and $\alpha$ is the change of the spin tune per radian of the orbit angle. When the beam is slowly $\left(\alpha \ll|\epsilon|^{2}\right)$ accelerated through the resonance, the spin vector will adiabatically follow the stable spin direction resulting in spin flip as is indicated in Fig. 2. However, for a faster acceleration rate partial depolarization or partial spin flip will occur.

Traditionally, the intrinsic resonances are overcome by using a betatron tune jump, which effectively makes $\alpha$ large, and the imperfection resonances are overcome with the harmonic corrections of the vertical orbit to reduce the resonance strength $\epsilon$ [4]. Both of these methods aim at making the resonance crossing nonadiabatic. They require very accurate adjustments at every resonance crossing which can become very difficult and time consuming. 


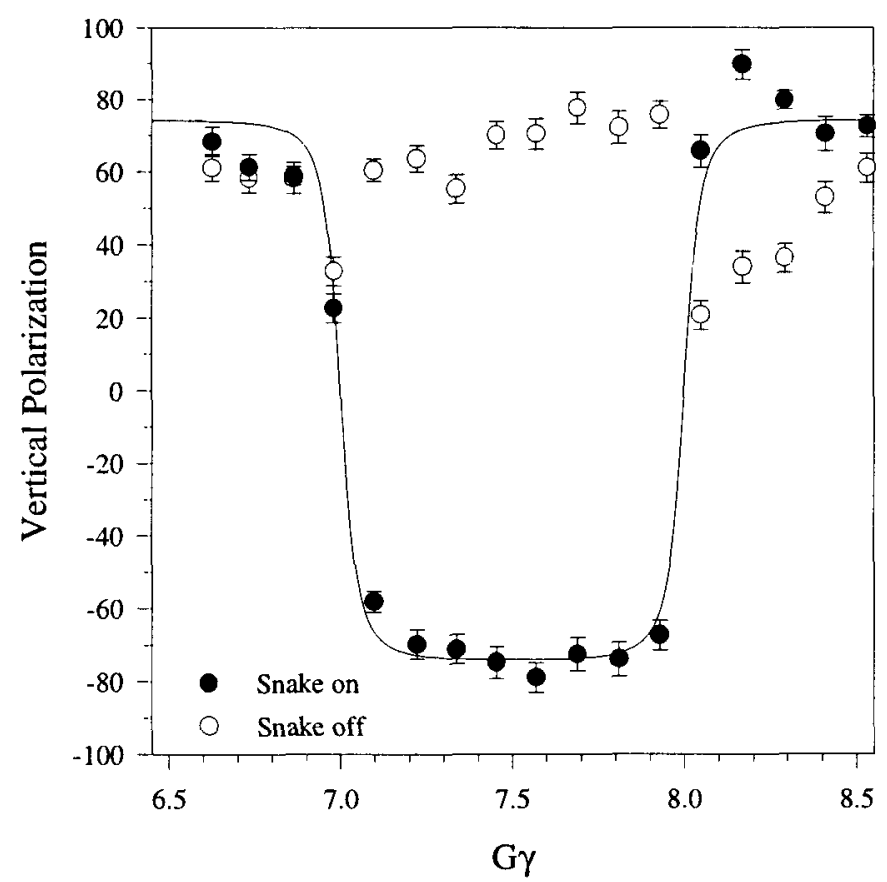

FIGURE 3. The measured vertical polarization as a function of $G \gamma$ for a $10 \%$ snake is shown with and without a snake. The solid line is the predicted energy dependence of the polarization.

Over the last ten years new techniques to cross both imperfection and intrinsic resonances adiabatically have been developed. The correction dipoles used to correct the imperfection resonance strength to zero were replaced by a localized spin rotator or 'partial Siberian snake' which makes all the imperfection resonance strengths large and causes complete adiabatic spin flip at every imperfection resonance [5]. The tune jump quadrupoles were recently replaced at the AGS by a single rf dipole magnet which creates a strong spin resonance by driving large coherent betatron oscillations, overpowering the effect of the intrinsic resonances.

At higher energies a 'full Siberian snake' [6], which is a $180^{\circ}$ spin rotator of the spin about a horizontal axis, will keep the stable spin direction unperturbed at all times as long as the spin rotation from the Siberian snake is much larger than the spin rotation due to the resonance driving fields. Therefore the beam polarization is preserved during acceleration. An alternative way to describe the effect of the Siberian snake comes from the observation that the spin tune with the snake is a half-integer and energy independent. Therefore, neither imperfection nor intrinsic resonance conditions can ever be met as long as the betatron tune is different from a half-integer.

A local spin rotator can be constructed by using either a solenoid at lower energies or at high energy by a sequence of interleaved horizontal and vertical dipole magnets producing only a local orbit distortion. Since the orbit distortion is inversely proportional to the momentum of the particle, such a dipole snake is particularly effective for high-energy accelerators, e.g. energies above about $30 \mathrm{GeV}$. 


\section{AGS Polarization}

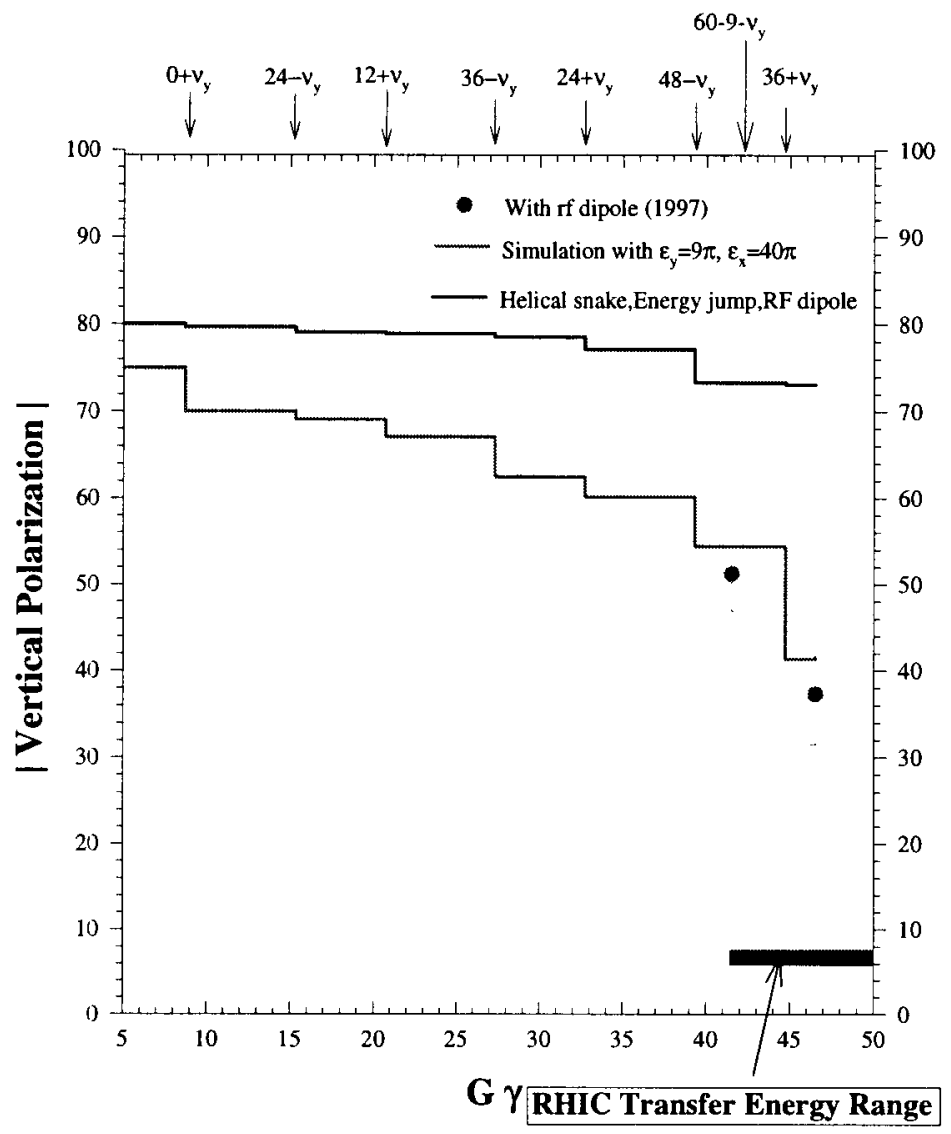

FIGURE 4. Vertical polarization versus $G \gamma$ measured in the AGS. The lower curve is the result of a spin tracking calculation for the experimental conditions. The upper curve simulates the use of a helical partial snake in the AGS.

\section{ACCELERATING POLARIZED PROTONS IN AGS AND RHIC}

Polarized proton beam experiments at the AGS have demonstrated the feasibility of polarized proton acceleration using a $5 \%$ partial Siberian snake. It was shown that a $5 \%$ snake is sufficient to avoid depolarization from imperfection resonances without using the harmonic correction method up to the required RHIC transfer energy of about $25 \mathrm{GeV}$. Fig. 3 shows the evolution of the beam polarization as the beam energy and therefore $G \gamma$ is increased [7]. As predicted the polarization reverses its sign whenever $G \gamma$ is equal to an integer. At this relatively low energy polarization is preserved even without snake but is partially lost at energies close to integer values of $G \gamma$. 
More recently a novel scheme of overcoming strong intrinsic resonances using a rf dipole magnet was successfully tested [9]. Full spin flip can be achieved with a strong artificial rf spin resonance excited coherently for the whole beam by driving large coherent vertical betatron oscillations. If the rf spin resonance location is chosen near the intrinsic spin resonance, the spin motion will be dominated by the rf resonance and the spin near the intrinsic resonance will adiabatically follow the spin closed orbit of the rf spin resonance. With the rf dipole, a new dominant resonance near the intrinsic resonance is introduced to flip the spin, instead of enhancing the intrinsic resonance, as has been proposed earlier [8], which would also enhance the strength of the nearby coupling resonance. Fig. 4 shows the new record proton beam polarization achieved during the last AGS polarized beam experiment. The rf dipole was used to completely flip the spin at the four strong intrinsic resonances $0+\nu_{y}, 12+\nu_{y}, 36-\nu_{y}$, and $36+\nu_{y}$. The lower curve shown going through the data points was obtained from a spin tracking calculation simulating the experimental conditions. Most of the remaining polarization loss is caused by the coupling resonances. A new AGS partial snake using a helical dipole magnet would eliminate all coupling resonances. Spin tracking simulations of this condition are depicted by the upper curve in Fig. 4.

With full snakes in RHIC all depolarizing resonances should be avoided since the spin tune is a half-integer independent of energy. However, if the spin disturbance from small horizontal fields is adding up sufficiently between the snakes depolarization can still occur. This is most pronounced when the spin rotation from all the focusing fields add up coherently which is the case at the strongest intrinsic resonances. A simplistic rule of thumb would then suggest that as long as the total spin rotation of all the Siberian snakes is much larger than the total spin rotation per turn caused by the strongest spin resonance the polarization should be preserved during acceleration. This rule holds for the AGS partial Siberian snake with regard to the imperfection resonances. It would also predict that for a beam with a normalized $95 \%$ emittance of $20 \pi \mathrm{mm} \mathrm{mrad}$ at least two snakes are needed for RHIC.

Polarized protons from the AGS are injected into the two RHIC rings to allow for up to $\sqrt{s}=500 \mathrm{GeV}$ collisions with both beams polarized [1]. Fig. 5 shows the layout of the Brookhaven accelerator complex highlighting the components required for polarized beam acceleration. A new polarized source using the 'Optically Pumped Polarized Ion Source' (OPPIS) technique is being assembled at TRIUMF using components of the KEK OPPIS [10]. The new source will be able to deliver $10^{12}$ polarized protons per pulse which will be injected into RHIC as a single bunch with nominally $2 \times 10^{11}$ polarized protons.

Of particular interest is the design of the Siberian snakes (two for each ring) and the spin rotators (four for each collider experiment) for RHIC. Each snake or spin rotator consists of four $2.4 \mathrm{~m}$ long, $4 T$ helical dipole magnet modules each having a full 360 degree helical twist [11]. Using helical magnets minimizes orbit excursions within the extend of the snake or spin rotator which is most important at injection energy. Nevertheless the bore of the helical magnets has to be $10 \mathrm{~cm}$ in diameter 


\section{Polarized Proton Collisions at BNL}

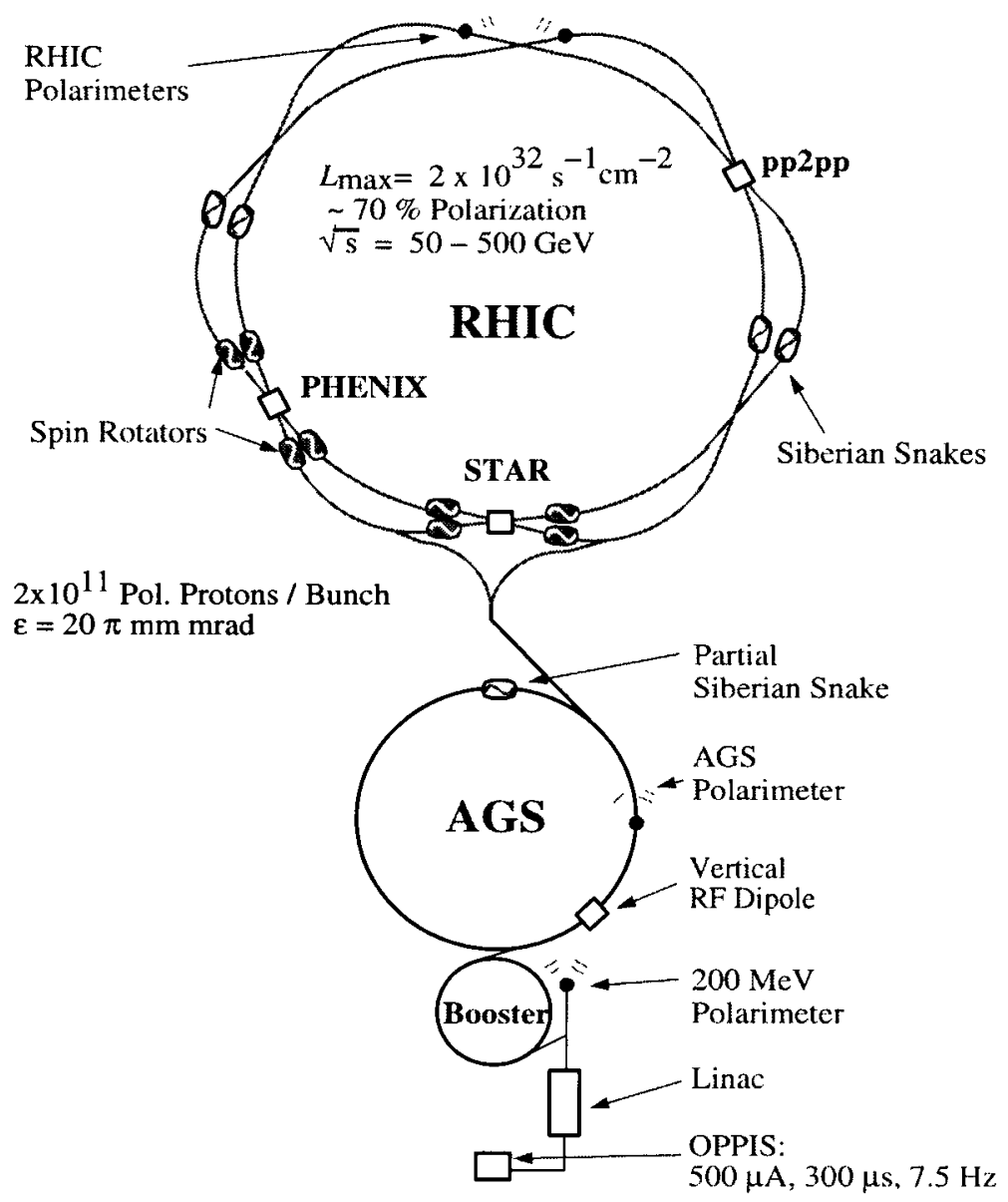

FIGURE 5. The Brookhaven hadron facility complex, which includes the AGS Booster, the AGS, and RHIC. The RHIC spin project will install two snakes per ring with four spin rotators per detector for achieving helicity-spin experiments.

to accommodate the $3 \mathrm{~cm}$ orbit excursions. Superconducting helical dipoles have been successfully tested at BNL using thin cable placed into helical grooves that have been milled into a thick-walled aluminum cylinder. A schematic picture of the helical dipole magnet is shown in Fig. 6. The first four full length magnets, enough for the first complete Siberian snake, have recently been successfully tested [12].

To verify that full polarization is preserved during acceleration in RHIC an elaborate spin tracking program was developed [13]. The acceleration through the energy region of the strongest resonance was simulated in great detail including a 1 $\mathrm{mm}$ rms misalignment of the quadrupoles, and sextupoles as well as the corrector dipoles used to correct the closed orbit. The result is shown in Fig. 7 for a beam with a normalized $95 \%$ emittance of $20 \pi \mathrm{mm}$ mrad. The upper and lower curve 


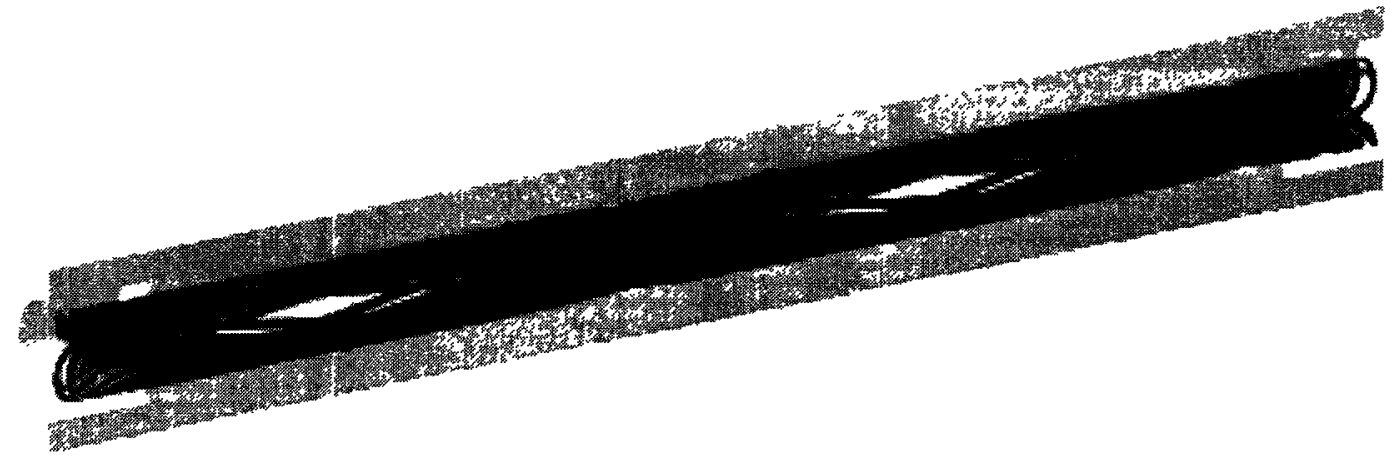

FIGURE 6. Schematic picture of the superconducting helical dipole shows the 16 helical current blocks and half of the iron yoke

show the result for the full beam and the particles at the edge of the beam, respectively. Although there is a significant decrease of the polarization at the energy of the resonance at $G \gamma=5 \times 81+\left(\nu_{y}-12\right)=422.18$, the polarization of the full beam is restored after accelerating completely through the resonance region. The simulation also shows that there is significant polarization loss at the edge of the beam. This fact highlights the need for a polarimeter that can measure polarization profiles.

Measuring proton polarization at high energies has been the focus of several workshops and experiments. The analyzing power of only very few reactions has been

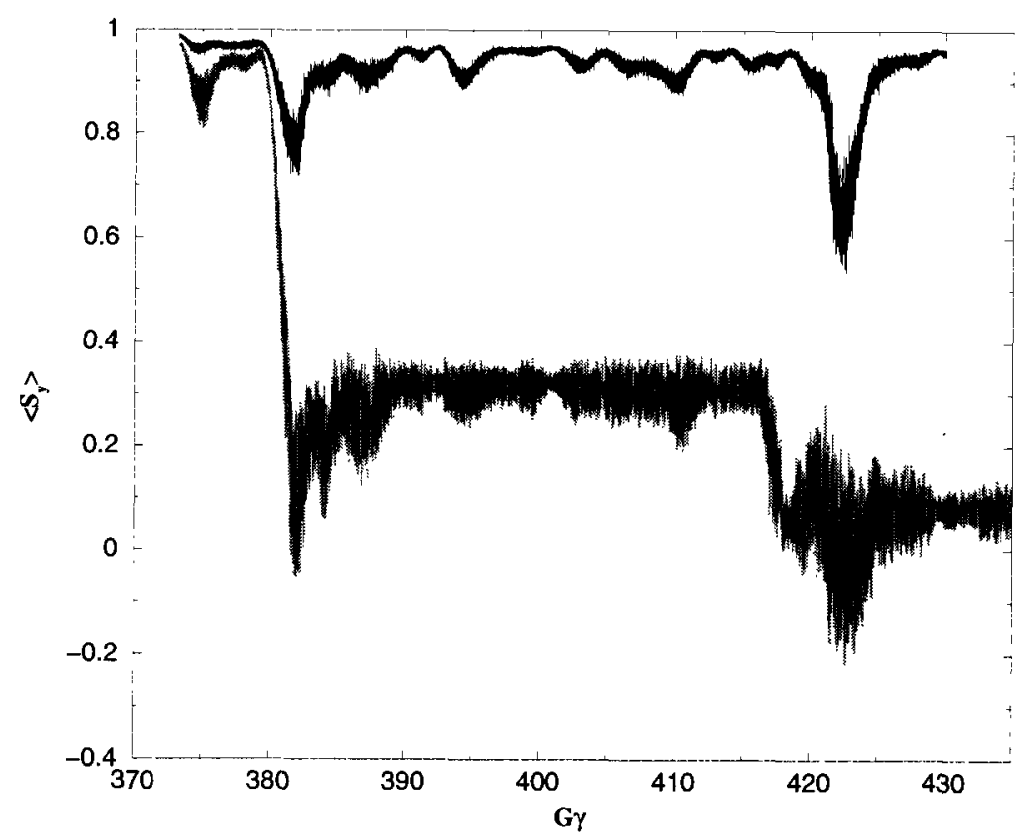

FIGURE 7. Result of spin tracking through the region of the strongest resonance in RHIC. The closed orbit was corrected with residuals of less than $0.2 \mathrm{~mm}$. 

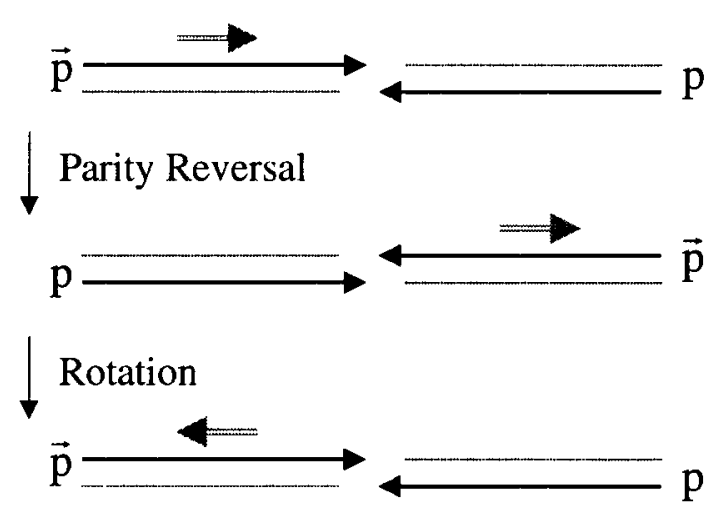

FIGURE 8. Schematic illustration of the implications of parity violation for the spin dependent proton-proton total cross section.

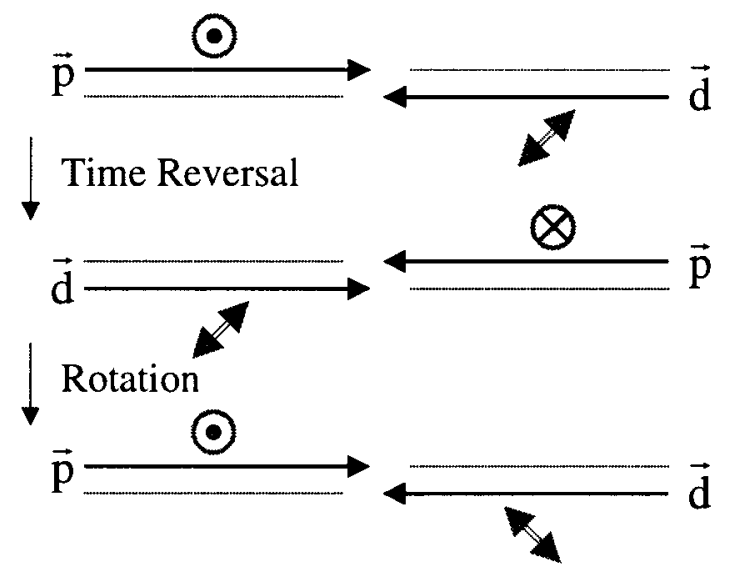

FIGURE 9. Schematic illustration of the implications of time reversal invariance for the spin dependent proton-deuteron total cross section.

measured at high energies and the magnitudes are typically rather small. Polarization sensitive interaction with an external electromagnetic field is also much smaller than for the much lighter electron for which Compton back scattering is typically used for high energy polarization measurement. Two methods are presently being considered for RHIC. Inclusive pion production from both hydrogen and carbon targets has been shown to have large analyzing power. Magnetic spectrometers are being designed for RHIC to allow for high rate data acquisition. The second method is based on the relatively large, energy independent analyzing power predicted for very small angle elastic scattering where the magnetic interaction interferes with the spin independent nuclear interaction [14]. Tests at the IUCF Cooler ring and with polarized beam at the AGS have demonstrated the possibility of detecting the recoil carbon nucleus from a ultra thin carbon fiber. A significant non-zero analyzing power at $22 \mathrm{GeV}$ was also measured. For both methods the carbon fiber target could be scanned through the circulating beam to measure polarization profiles.

\section{SYMMETRY TESTS AT RHIC}

The polarized proton beams in RHIC can also be used with fixed internal targets. This offers the unique opportunity to study parity violation and violation of time reversal invariance in total cross section measurements over a very large energy range. As is shown schematically in Fig. 8 measuring a difference of the total cross section for the two helicity states of the beam would violate parity. This could be accomplished with high statistical accuracy by simply measuring the difference in the beam life time between the two helicity states [15]. A similar scheme can be used to measure the violation of time reversal invariance by using transverse polarized proton beam and a tensor-polarized deuterium gas target [16]. Such measurements 
pose a tremendous demand on the detailed understanding of systematic errors but the unique and novel environment of a storage ring may make such high precession experiments possible.

\section{COMMISSIONING PLANS AND SCHEDULE}

It is planned that, for the expected turn-on of RHIC in 2000 , one ring will be equipped with two snakes and a polarimeter which will allow for initial commissioning of polarized beam acceleration. Starting after October 2000 all snakes and spin rotators will be in place for the first RHIC spin physics run with longitudinal polarization at the two detectors STAR and PHENIX.

\section{REFERENCES}

1. Design Manual - Polarized Proton Collider at RHIC, Brookhaven National Laboratory, July 1998, http://www.ags.bnl.gov/ rhicspin .

2. L.H. Thomas, Phil. Mag. 3, 1 (1927); V. Bargmann, L. Michel, V.L. Telegdi, Phys. Rev. Lett. 2, 435 (1959).

3. M. Froissart and R. Stora, Nucl. Instr. Meth., 1, 297 (1960).

4. T. Khoe et al., Part. Accel. 6, 213 (1975); J.L. Laclare et al., J. Phys. (Paris), Colloq. 46, C2-499 (1985); H. Sato et al., Nucl. Inst. Meth., Phys. Res. Sec A272, 617 (1988); F.Z. Khiari, et al., Phys. Rev. D39, 45 (1989).

5. T. Roser, AIP Conf. Proc. No. 187, ed. K.J. Heller p.1442 (AIP, New York, 1988).

6. Ya.S. Derbenev et al., Part. Accel. 8, 115 (1978).

7. H. Huang et al., Phys. Rev. Lett. 73, 2982 (1994)

8. T. Roser, in Proc. of the 10th Int. Symp. on High Energy Spin Physics, Nagoya, Japan, p. 429 (1992).

9. M. Bai et al., Phys. Rev. Lett. 80, 4673 (1998)

10. A.N. Zelinski et al., 'Optically-Pumped Polarized H- ION Sources for RHIC and HERA Colliders', Proceedings of the 1999 Particle Accelerator Conference, to be published.

11. V.I.Ptitsin and Yu.M.Shatunov, Helical Spin Rotators and Snakes, Proc. 3. Workshop on Siberian Snakes and Spin Rotators (A.Luccio and T.Roser Eds.) Upton, NY, Sept. 12-13,1994, BNL-52453, p.15;

12. E. Willen et al., 'Construction of helical magnets for RHIC', Proceedings of the 1999 Particle Accelerator Conference, to be published.

13. A.U. Luccio et al., 'Development of the spin tracking program SPINK', Proceedings of the 1999 Particle Accelerator Conference, to be published.

14. J. Schwinger, Phys. Rev. 73, 407 (1948)

15. S.E. Vigdor, in Proc. Workshop on Future Directions in Particle and Nucl. Physics (D.F. Geesaman, Ed.), Brookhaven 1993, Rep. BNL-52389, 1993, p.173

16. H.E. Conzett, Phys. Rev. C48, 423 (1993) 